\title{
Stress hyperglycemia and electrolytes disturbance in patients with acute cerebrovascular stroke
}

\author{
Bastawy M. El-Fawal ${ }^{1 *}$, Reda Badry², Wael A. Abbas ${ }^{3}$ and Ahmed K. Ibrahim ${ }^{4}$
}

\begin{abstract}
Background: Stroke is a medical emergency and considered the 2nd leading cause of mortality worldwide. High morbidity and mortality of stroke are due to some complications like cerebral edema, infection, and associated heart disease. Stroke may cause stress hyperglycemia and electrolyte disturbance that may be independent predictors for increased morbidity and mortality. Data on electrolyte disorder in neurological conditions especially acute stroke are somewhat scanty and not frequently interpreted.
\end{abstract}

Objectives: The current study aimed to evaluate the effect of stress induced by acute stroke on the blood glucose and serum electrolytes and its relationship to the type, severity, and prognosis of stroke.

Subjects and methods: A total of 100 patients admitted with acute stroke were included in the study. After informed consent, fasting blood sugar, glycosylated hemoglobin, serum sodium, and potassium levels were measured simultaneously. Stroke severity and outcome were assessed using the Glasgow Coma Scale.

Results: Stress hyperglycemia was noted in 21 patients, out of which $62 \%$ presented with hemorrhagic stroke. Undiagnosed diabetes was found in $47 \%$ of cases. Also, $13 \%$ of our patients had both stress hyperglycemia and electrolyte disturbance simultaneously. Mortality among hemorrhagic versus ischemic patients was $46 \%$ and $22 \%$, respectively. The independent predictors of mortality were stress hyperglycemia, electrolytes disturbance, ischemic heart diseases, and type and admission severity of stroke.

Conclusion: Stress hyperglycemia and electrolyte disturbance after acute stroke were found in $21 \%$ and $47 \%$ of patients respectively and may adversely affect outcome of stroke especially those with cerebral hemorrhage.

Keywords: Stress hyperglycemia, Electrolyte disturbance, Stroke

\section{Introduction}

According to the WHO, cerebrovascular stroke (CVS) is the rapidly developing loss of brain functions due to a disturbance in the blood supply to the brain, lasting more than $24 \mathrm{~h}$ or interrupted by death with no apparent cause other than of vascular origin. Moreover, it is a medical emergency that can cause permanent neurological damage, complications, disability, and death [1]. CVS is considered as one of the stressors that may cause stress hyperglycemia and/or electrolyte disturbance [2]. Hyperglycemia is a common problem in stroke patients,

\footnotetext{
* Correspondence: s_monur@yahoo.com

${ }^{1}$ Neuropsychiatry Department Faculty of Medicine, Aswan University, Aswan, Egypt

Full list of author information is available at the end of the article
}

and it is estimated that $20-50 \%$ of acute stroke patients presented with concurrent diagnosis of hyperglycemia which has been linked to poor prognosis [3]. The development of stress hyperglycemia is caused by a highly complex interplay of counter-regulatory hormones such as catecholamines, growth hormone, cortisol, and cytokines and the mechanism vary with the patients' underlying glucose tolerance, type and severity of disease, and stage of illness [4]. Acute hyperglycemia is associated with increased edema and infarct size and with reduced cerebral blood flow and cerebrovascular reserve, and it can increase the ischemic brain damage around an intracerebral hemorrhage [3]. Disorders of sodium and potassium concentration are the commonest electrolyte abnormalities found in stroke patients, resulted from 
inappropriate secretion of antidiuretic hormones, increase in the brain and atrial natriuretic peptides, and inappropriate fluid intake and loss; this can lead to complications like seizures and death [5]. Most hemorrhagic stroke cases presented with headache and vomiting, which is an important cause of electrolyte disturbance. Thus, early diagnosis of electrolyte disturbance is essential to prevent morbidity and mortality of CVS patients. However, data on electrolyte disturbances are still scanty [6]. Therefore, the current study aimed to evaluate the effect of stress induced by acute CVS on blood glucose and electrolyte balance and its relationship to the type of stroke, severity, and prognosis (Fig. 1).

\section{Patients and methods}

The current work was a case-comparison study. Sixty male and 40 female patients with acute CVS (within 1 week) who were admitted to stroke care monitoring unit and internal medicine department of Aswan University Hospital from July to December 2018 were included in the current study. Patients proved by CT (Siemens Spirit Dual Slice CT Scanner, Rs 54 Lakh, Germany) to have brain pathology other than stroke, refuse to participate, known to be diabetic, renal, hepatic, and those receiving medications that promote electrolyte disturbance "fluids, furosemide, corticosteroids, and thiazides" were excluded.

Patients were classified according to their clinical picture and investigations including CT (Siemens Spirit Dual Slice CT Scanner, Rs 54 Lakh, Germany) and/or MRI brain (Siemens Trio 3 T MRI Scanner, Rs 385 Lakh, Germany) into ischemic and hemorrhagic stroke (cerebral and subarachnoid hemorrhage).

Sample size calculation was carried out using G*Power 3 software [7]. A calculated minimum sample of 98 stroke patients (raised to 100) divided into two groups (50 hemorrhagic and 50 ischemic) were needed to detect an effect size of 0.1 in the mean Glasgow Coma Scale (GCS), with an error probability of 0.05 and $90 \%$ power on a two-tailed test.

Data on self or family reported risk factors for stroke, including socio-demographics, hypertension, heart diseases, and smoking, were obtained from the patient's medical records. Fasting blood glucose level was measured glucometer (Accu Check Advantage ${ }^{\circ}$, Roche, USA) after hospital admission, and increased glucose level was considered when fasting blood glucose level was $>110$ $\mathrm{mg} / \mathrm{dl}$. Glycosylated hemoglobin (HbA1c) was measured by test kits (Eurolyser * Eurolyser Diagnostica GmbH, Bindergasse 3, Salzburg/Austria) to rule out undetected diabetic patients (normal value $4.5-<6.5 \%$, while HbA1c $\geq 6.5 \%$ means previously uncontrolled hyper glycemia). Stress hyperglycemia was labeled if fasting blood glucose $>110 \mathrm{mg} / \mathrm{dl}$ together with HbA1c $<6.5 \%$, and undiagnosed diabetes was diagnosed when $\mathrm{HbA} 1 \mathrm{c} \geq 6.5 \%$ of blood sugar. Serum sodium $(\mathrm{Na})$ was measured by MODULAR ANALYTICS E170 ${ }^{\circ}$ system (Roche Diagnostics, Indianapolis, IN) for all patients (normal $\mathrm{Na}=$ 135-145 mEq/L), where, $\mathrm{Na}>145 \mathrm{mEq} / \mathrm{L}$ indicates hypernatremia [8], whereas $\mathrm{Na}<135 \mathrm{mEq} / \mathrm{L}$ indicates hyponatremia [9]. Also, serum potassium (K) was measured (normal $K=3.5-5 \mathrm{mEq} / \mathrm{L}$ ), where, $K>5 \mathrm{mEq} / \mathrm{L}$ means hyperkalemia and $K<3.5 \mathrm{mEq} / \mathrm{L}$ means hypokalemia [10]. A standard 12-leads electrocardiogram (ECG) and/or echocardiography were performed to all stroke patients to determine any arrhythmias and/or any ischemic changes (CON-TEC, model: ECG100G, China).

Initial assessment of CVS severity at time of admission and follow up-assessment after 1 week by GCS and the number of deaths if present were estimated. GCS is of

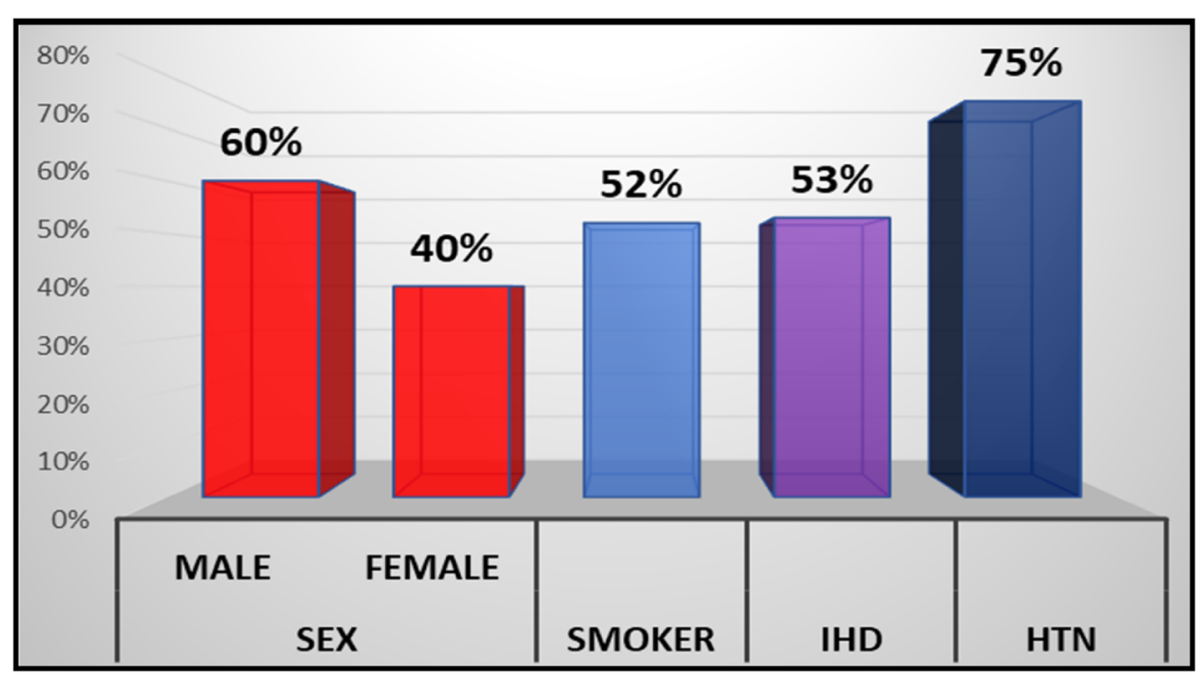

Fig. 1 Basic data of cerebrovascular stroke patients 
prognestic value in acute CVS patients and is widely used in assessing level of consciousness [11], which may the mostly affected with stress hyperglycemia and electrolyte disturbance, while other scales such as the National Institutes Of Health Stroke Scale (NIHSS) evaluate the outcome of therapeutic management of CVS patients (not fessible to our patients due to many obstacles as, lack of finance, arrival after time window for stoke). Moreover, we used Barthel Index [12], but the results were insignificant. Generally, brain injury is classified as severe (GCS $\leq 8$ ), moderate (GCS 9-12), and minor $(\mathrm{GCS} \geq 13)$ [13].

\section{Statistical analysis}

Data were verified, coded by the researcher and analyzed using IBM-SPSS version-21 (@IBM-SPSS Inc., 2012, NY-USA) [14]. Descriptive statistics means, standard deviations, and percentages were calculated. Test of significances chi-square test was used to compare the difference in distribution of frequencies among different groups. The clinical and demographic factors with proven statistical significance from the univariate analyses were further included in the multivariate logistic regression model. A $p$ value equals or less than 0.05 was considered significant.

\section{Ethical consideration}

Approval for this study was obtained from the institutional review board (IRB) of Faculty of Medicine Aswan University prior to study execution. In addition, all participants' caregivers received a written consent form. The informed consent was clear and indicated the purpose of the study, and their freedom to participate or withdraw at any time without any obligation. Furthermore, participants' confidentiality and anonymity were assured by assigning each participant with a code number for the purpose of analysis only. The study was not based on any incentives or rewards for the participants.

\section{Results}

A total of 100 patients admitted with CVS to stroke care monitoring unit and internal medicine department of Aswan University Hospital from July to December 2018 (50 patients for each type of stroke) were evaluated. The mean age of them was $64 \pm 12$ years, and we found $52 \%$ smokers, $53 \%$, and $75 \%$ of ischemic heart diseases and hypertensive respectively (Table 1 ).

About two thirds of the studied patients had hyperglycemia ( $70 \%$ of hemorrhagic patients), out of them, 47 patients had underlying undiagnosed diabetes and the remaining $21 \%$ had stress hyperglycemia (13 hemorrhagic versus 8 ischemic patients). Moreover, $47 \%$ of all stroke patients had dyselectrolytemia without any significant difference between the two stroke types $(p=$
Table 1 Basic data of cerebrovascular stroke patients

\begin{tabular}{lll}
\hline Age/years (mean \pm SD) & $64.4 \pm 12.1$ & \\
Sex & & $60 \%$ \\
$\quad$ Male & 60 & $40 \%$ \\
Female & 40 & $52 \%$ \\
Smoking & 52 & $53 \%$ \\
Ischemic heart diseases & 53 & $75 \%$ \\
Hypertension & 75 & \\
\hline
\end{tabular}

0.163). Hyponatremia was the most frequently encountered dyselectrolytemia (in 36 patients, out of them 21 hemorrhagic and 15 ischemic patients), followed by hyperkalemia (in 27 patients, 17 of which had cerebral hemorrhage) and lastly hypokalemia which was found in 8 of all patients that distributed equally among the two groups of patients (Table 2).

According to GCS which is used to evaluate the stroke severity, about one third of the studied cohort had severe disability. While moderate disability was detected in $8 \%$ of our patients and 58 patients had good recovery, out of which, only 4 patients had stress hyperglycemia (7\%) that was detected in $44 \%$ of those with poor prognosis (15 patients). Among dyselectrolytemic, patients with good outcome were found in 30\% of cases and about two thirds of cases were with poor prognosis. According to the type of dyselectrolytemia, poor outcome was detected in $67 \%, 75 \%$, and $78 \%$ of hyponatremic, hypokalemic, and hyperkalemic patients respectively (Table 3).

In this present study, $34 \%$ of all patients died, and nearly two thirds of them were hemorrhagic. Death was recorded in $71 \%(n=15 / 21)$ and $66 \%(n=31 / 47)$ of patients who had stress hyperglycemia and dyselectrolytemia, respectively. Furthermore, death rate reached 100\% in patients who had both stress hyperglycemia and dyselectrolytemia simultaneously (13 patients). Death rate was $67 \%(n=24 / 36), 75 \%(n=6 / 8)$, and $78 \%(n=21 /$ 27) in hyponatremic, hypokalemic, and hyperkalemic patients, respectively (Table 4).

Table 5 showed the multivariate logistic regression analysis of the significant factors affecting mortality of stroke patients. After adjusting for age and sex, the final model contained five predictors. Patients with hemorrhagic stroke were three times more at risk compared with ischemic cases [AOR $=3.02(1.27-7.21), p=$ 0.013]. Likewise, patients with stress hyperglycemia had eight times more probability of death compared with cases with normal blood glucose level [AOR $=7.9(2.7-$ 23.2), $p<0.001]$.

Moreover, patients with electrolyte disturbance were 10.8 times more liable for mortality compared with cases with normal electrolyte level $[\mathrm{AOR}=10.8(4.11-28.41)$, 
Table 2 Laboratory data differences between types of stroke

\begin{tabular}{llll}
\hline Variable & $\begin{array}{l}\text { Hemorrhagic } \\
(n=50)\end{array}$ & $\begin{array}{l}\text { Ischemic } \\
(n=50)\end{array}$ & $P$ value $^{\mathrm{a}}$ \\
\hline Diabetic status & & $33(66 \%)$ & $=0.415$ \\
Hyperglycemia & $35(70 \%)$ & $25(50 \%)$ & $=0.548$ \\
Undiagnosed DM & $22(44 \%)$ & $8(16 \%)$ & $=0.163$ \\
Stress hyperglycemia & $13(26 \%)$ & $22(44 \%)$ & $=0.163$ \\
Electrolytes disturbance & $25(50 \%)$ & $15(30 \%)$ & $=0.149$ \\
Hyponatremia & $21(42 \%)$ & $10(20 \%)$ & $=0.214$ \\
Hyperkaliemia & $17(34 \%)$ & $4(8 \%)$ & $=0.214$ \\
Hypokalemia & $4(8 \%)$ &
\end{tabular}

${ }^{a}$ Chi-square test was used to compare the proportions among groups. DM diabetes mellitus

$p<0.001]$. Also, with one-point increase in GCS, there was $58 \%$ decrease in the risk of mortality, and this was statistically significant $(p<0.001)$. Finally, ischemic heart disease (IHD) patients had 3.8 times more risk of death [AOR $=3.77$ (1.53-9.32), $p=0.004]$.

\section{Discussion}

High blood pressure, old age, smoking, and cardiac diseases were established as independent correlates for CVS and results of the current study supported this fact. Hyperglycemia is common among acute CVS patients and may reflect a physiological stress or an underlying unrecognized diabetes mellitus (DM, [15]). It was associated with in-hospital and long-term mortality, intensive care unit admission, prolonged length of stay, and discharge to long-term nursing $[16,17]$. Our study evaluated stress induced hyperglycemia in patients with acute CVS and found that about one-fifth of patients had hyperglycemia in the absence of DM. This result was comparable to other studies [18, 19]. Likewise, Capes et al., 2001, we observed that stress hyperglycemia was more common among patients with hemorrhagic than ischemic stroke $(p$ value $=0.163)$. Overall, about half of our stroke patients (47\%) had undiagnosed diabetes at the time of admission, which was higher than the range that stated by Capes et al., 2001, from 6 to $42 \%$ of patients [20]. This may be explained by reluctance of people in our community to check their medical condition regularly. The current study findings confirmed the conclusions of previous studies [21, 22] that acute CVS is not uncommonly associated with electrolyte imbalance and hyponatremia was the commonest but the association between type of stroke and dyselectrolytemia was statistically insignificant $(p=0.163)$. Furthermore, the percentage of potassium imbalance (35\%) was higher than other studies [21, 23], and this may be explained by associated hyperglycemia (in $68 \%$ of cases) that increases release of potassium from cells.

Thirty-four percent of our CVS patients had poor outcome, which was comparable to that of Maitreyee et al., 2017 (37\%), this poor outcome was detected more among hemorrhagic patients and those with severe initial stroke, IHD, stress hyperglycemia (70\%), and electrolyte disturbance (66\%). The percentage of our poor outcome was higher than other studies [21, 24] may as a result of non-specific treatment used with stroke patients. Percentages of poor outcome and death (34\% for each) were consistent with those of World Health Organization (WHO), where about 15 million people suffer stroke worldwide every year, out of which 5 million die and 5 million are permanently disabled [25]. Likewise, other studies [26], the rate of mortality among our hemorrhagic stroke patients was more than double that of ischemic patients ( $46 \%$ vs. $22 \%$, respectively). Finding of death among $71 \%$ of stress induced hyperglycemic patients was supported by many studies [27-29], which reported that hyperglycemia had a deleterious effect, exacerbating brain injury, increasing the relative risk of death by three times and is considered a significant predictor of mortality and morbidity. About two thirds of patients with dyselectrolytemia died, this was higher than those detected by other studies [21, 24, 30], and this could be explained by the underdevelopment of the acute management in our

Table 3 Distribution of outcome of stroke patients according to the GCS after a week

\begin{tabular}{|c|c|c|c|c|}
\hline Variable & Good outcome $(n=58)$ & Moderate outcome $(n=8)$ & Poor outcome $(n=34)$ & $P$ value \\
\hline Hemorrhagic $(n=50)$ & $23(46 \%)$ & $4(8 \%)$ & $23(46 \%)$ & $=0.010$ \\
\hline Ischemic $(n=50)$ & $35(70 \%)$ & $4(8 \%)$ & $11(22 \%)$ & \\
\hline Stress hyperglycemia $(n=21)$ & $4(19.1 \%)$ & $2(9.5 \%)$ & $15(71.4 \%)$ & $<0.001$ \\
\hline Electrolytes disturbance $(n=47)$ & $14(29.7 \%)$ & $2(4.3 \%)$ & $31(66 \%)$ & $<0.001$ \\
\hline Hyponatremia $(n=36)$ & $10(27.8 \%)$ & $2(5.6 \%)$ & $24(66.6 \%)$ & $<0.001$ \\
\hline Hyperkalemia $(n=27)$ & $6(22.2 \%)$ & $0(0 \%)$ & $21(77.8 \%)$ & $=0.002$ \\
\hline Hypokalemia $(n=8)$ & $2(25 \%)$ & $0(0 \%)$ & $6(75 \%)$ & $=0.002$ \\
\hline
\end{tabular}

${ }^{\mathrm{a}}$ Chi-square test was used to compare the proportions among groups 
Table 4 Distribution of stroke patients according to the mortality

\begin{tabular}{lll}
\hline Variable & Total no. $=$ & $(\%)$ \\
\hline Stroke type & 34 & \\
$\quad$ Hemorrhagic $(n=50)$ & 23 & 46 \\
$\quad$ Ischemic $(n=50)$ & 11 & 22 \\
Stress hyperglycemia $(n=21)$ & 15 & 71.4 \\
Electrolyte disturbance $(n=47)$ & 31 & 66 \\
Hyponatremia $(n=36)$ & 24 & 66.7 \\
Hypokalemia $(n=8)$ & 6 & 75 \\
$\quad$ Hyperkalemia $(n=27)$ & 21 & 77.8 \\
Both stress hyperglycemia and electrolyte & 13 & 100 \\
disturbance $(n=13)$ & & \\
\hline
\end{tabular}

hospital. Huang et al., 2012 [31] found that hyponatremia in acute stroke stage is a predictor for 3-year mortality, and we detected death in about two thirds of hyponatremic stroke patients which was higher than Maitreyee et al. (45\%). In the CASTEL study, hyponatremia has been found to be a predictor of CVS mortality with relative risk of 1.34 and hyperkalemia was also identified as a risk factor [32]. Also, Gariballa et al. found that a lower plasma potassium level on admission was associated with an increased incidence of death. More than three quarters of our patients with potassium imbalance died; this was higher than other studies $[21,33]$. Augmentation of the deleterious effects of stress-induced hyperglycemia and electrolyte disturbance led to death of all stoke patients who had both problems at the same time.

In conclusion, stress-related hyperglycemia and electrolyte disturbance are quite common problems after acute stroke and may adversely affect outcome of stroke. So, blood sugar and serum electrolytes should be determined in every patient with stroke, as early detection and management can decrease complications and improve the overall outcome. Further larger studies are recommended with determination of causes of electrolyte disturbance to evaluate and compare the effect of different degrees of stress hyperglycemia and electrolyte disturbance on outcome of CVS.

Table $\mathbf{5}$ Independent correlates of mortality: multivariate regression analysis

\begin{tabular}{llll}
\hline Factor & Adjusted OR & $95 \% \mathrm{Cl}$ & LRT $P$ value \\
\cline { 2 - 4 } Stroke type (hemorrhagic) & 3.020 & $1.265-7.209$ & $=0.013$ \\
Stress hyperglycemia & 7.895 & $2.686-23.206$ & $<0.001$ \\
Electrolyte disturbance & 10.8 & $4.106-28.41$ & $<0.001$ \\
GCS at baseline & 0.416 & $0.297-0.584$ & $<0.001$ \\
Ischemic heart diseases & 3.77 & $1.525-9.317$ & $=0.004$ \\
\hline
\end{tabular}

Cl confidence interval, $O R$ odds ratio, $L R T$ likelihood ratio test

\section{Limitation of the study}

The results of the present study should be interpreted in the light of the following limitations. First, the sample size was relatively small. Second, our CVS patients received non-specific treatment with short-term follow-up (1 week) that caused more valuable prognostic scales (NIHSS and Barthel Index) yielded insignificant results. Third, the definite causes of electrolyte disturbance during acute CVS could not be carried out.

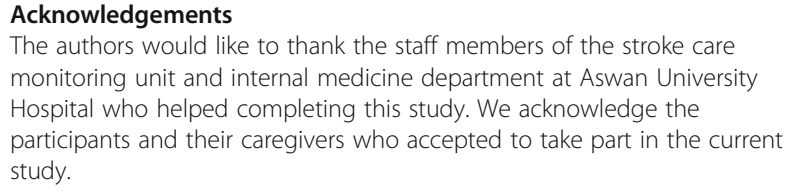

\section{Authors' contributions}

BF contributed to the concept, design, literature search, clinical studies, experimental studies, manuscript preparation, editing, and review of the study. RB contributed to the definition of intellectual content, literature search, and manuscript review. WA contributed to the clinical studies, experimental studies, and data acquisition. AKI contributed to the design, data analysis, statistical analysis, manuscript preparation, editing, and review of the manuscript. All authors read and approved the final study.

\section{Funding}

Not applicable

\section{Availability of data and materials \\ The datasets generated and/or analyzed during the current study are not publicly available due [Ethical issues] but are available from the corresponding author on reasonable request.}

\section{Ethics approval and consent to participate}

Approval for this study was obtained from institutional review board (IRB) of Faculty of Medicine Aswan University prior to study execution (No. 21, Jan. 2018). In addition, all participants' caregivers received a written consent form. The informed consent was clear and indicated the purpose of the study, and their freedom to participate or withdraw at any time without any obligation. Furthermore, participants' confidentiality and anonymity were assured by assigning each participant with a code number for the purpose of analysis only. The study was not based on any incentives or rewards for the participants.

\section{Consent for publication}

Not applicable

\section{Competing interests}

All authors declare that they have no competing interests.

\section{Author details}

${ }^{1}$ Neuropsychiatry Department Faculty of Medicine, Aswan University, Aswan, Egypt. ${ }^{2}$ Neuropsychiatry Department, Faculty of Medicine, Assiut University, Assiut, Egypt. ${ }^{3}$ Community Medicine Department, Assiut University, Assiut, Egypt. ${ }^{4}$ Internal Medicine Department, Assiut University, Assiut, Egypt.

Received: 24 May 2019 Accepted: 2 December 2019

Published online: 17 December 2019

\section{References}

1. Donnan G, Fisher M, Macleod M. Stroke. Lancet. 2008;371(9624):1612-23.

2. Davel J, Engel M, Freercks R, Peter J, May W, Badri M, et al. Abnormal glucose metabolism in non-diabetic patients presenting with an acute stroke: prospective study and systematic review. QJM. 2010;103(7):495-503.

3. Samiullah S, Qasim R, Imran S. Frequency of stress hyperglycaemia and its' influence on the outcome of patients with spontaneous intracerebral haemorrhage. JPMA. 2010;60(8):660-3. 
4. Barth E, Albuszies G, Baumgart K. Glucose mechanism and catecholamines. Crit Care Med. 2007;35(suppl):508-18.

5. Roy K, Bandyopadhyay R, Paul R, Chakraborty S, Ray D, Mitra S, et al. Study on serum and urinary electrolyte changes in cerebrovascular accident. JACM. 2014;15(2):91-5

6. Kusuda K, Saku Y, Sadoshima S. Disturbances of fluid and electrolyte balance in patients with acute stroke. Japanese J Geriatr. 1989;26(3):223-7.

7. Faul F, Erdfelder E, Lang A. A. B. G*Power 3: a flexible statistical power analysis program for the social, behavioral, and biomedical sciences. Behav Res Methods. 2007;39(1):175-91.

8. Muhsin SA, Mount DB. Diagnosis and treatment of hypernatremia. Best Pract Res Clin Endocrinol Metab. 2016;30(2):189-203.

9. Hoorn EJ, Zietse R. Diagnosis and treatment of hyponatremia: complication of the guidelines. J Am Soc Nephrol. 2017;28(5):1340-9.

10. Vanholder R, Van Biesen W, Nagler EV. Treating potassium disturbances: kill the killers but avoid overkill. Acta Clinica Belgica. 2019;74(4):215-28.

11. Weir CJ, Bradford AP, Lees KR. The prognostic value of the components of the Glasgow Coma Scale following acute stroke. Qjm. 2003;96(1):67-74.

12. Mahoney F, Barthel D. Functional evaluation: the Barthel Index. Md Med J. 1965;14:61-5.

13. Meredith W, Rutledge R, Fakhry S. The con undrum of the Glasgow Coma Scale in intubated patients: a linear regression prediction of the Glasgow verbal score from the Glasgow eye and motor scores. J Trauma. 1998;28(1):13-6.

14. IBM_SPSS. Statistical Package for Social Science. NY-USA: @IBM-SPSS Inc:; 2012.

15. Cardino M, Josol C, Manalo G. Prevalence and outcomes of unrecognized diabetes mellitus and prediabetes among acute stroke patients with admission hyperglycemia at the Philippine General Hospital: DASH Study. Phil J Int Med. 2011;49(2):79-87.

16. Kosiborod M, Rathore S, Inzucchi S, Masoudi F, Wang Y, Havranek E, et al. Admission glucose and mortality in elderly patients hospitalized with acute myocardial infarction: implications for patients with and without recognized diabete. Circ. 2005;111(23):3078-86

17. Matz K, Keresztes K, Tatschl C, Nowotny M, Dachenhausen A, Brainin M, et al. Disorders of glucose metabolism in acute stroke patients: an underrecognized problem. Diabetes Care. 2006;29(4):792-7.

18. Al-himyari F, Abbas F. Stress hyperglycemia in non diabetic Iraqi patients presenting with acute stroke. Endocr Pract. 2007;13(6):691-2.

19. Levetan C. Effect of hyperglycemia on stroke outcomes. Endocrinal Pract. 2004;10(Suppl 2):34-9.

20. Capes S, Hunt D, Malmberg K, Pathak P, Gerstein H. Stress hyperglycemia and prognosis of stroke in nondiabetic and diabetic patients: a systematic overview. Stroke. 2001;32(10):2426-32.

21. Maitreyee B, Sanat K, Mihir A, Anindyashankar B. Study of electrolyte abnormality in acute stroke. Ann Int Med Den Res. 2017;3(5):ME04-ME9.

22. Meenakshi K, Waleed K, Kanitkar S, Abhijit M, Rajdeb S. Electrolyte imbalance in acute stroke. NJIRM. 2017;8(4):23-6.

23. Song E, Chu K, Jeong S, Jung K, Kim S, Kim M, et al. Hyperglycemia exacerbates brain edema and perihematomal cell death after intracerebral hemorrhage. Stroke. 2003;34(1):221-5.

24. Butungeshwar $\mathrm{P}$, Chakradhar M, Sunil K. Clinical profiles, electrolytes status in acute strokes and their outcome. Int J Adv Med. 2018;5(3):492-7.

25. World Health Organization. The top 10 causes of deaths. 2014; Available at http://www.who.int/mediacentre/factsheets/fs310/en. Accessed 18 Feb 2019.

26. Michael A, Spinler S, Leeper B, Ohman E, Alexander K, Newby L, et al. Medication errors in acute cardiovascular and stroke patients: a scientific statement from the American Heart Association. Circulation. 2010;121(14): 1664-82.

27. Bruno A, Biller J, Adams H, Clarke W, Woolson R, Williams L, et al. Trial of ORG 10172 in acute stroke treatment (TOAST) investigators: acute blood glucose level and outcome from ischemic stroke. Neurology. 1999;52(2):280-4.

28. Moulin $T$, Tatu $L$, Crépin-Leblond $T$, Chavot $D$, Bergès $S$, Rumbach $T$. The Besancon stroke Registry: an acute stroke registry of 2500 consecutive patients. Eur Neurol. 1997;38(1):10-20.

29. Toni D, De Michele M, Fiorelli M, Bastianello S, Camerlingo M, Sacchetti M, et al. Influence of hyperglycaemia on infarct size and clinical outcome of acute ischemic stroke patients with intracranial arterial occlusion. J Neurol Sci. 1994;123(1-2):129-33.

30. Zhenwei G, Tianzhu W, Zhang J. Clinical analysis of electrolyte imbalance in thalamic hemorrhage patients within 24 hs. after admission. Acta Neurochir Suppl. 2011;111(4):343-48.
31. Huang W, Weng W, Peng T, Chien Y, Wu C, Lee M, et al. Association of hyponatremia in acute stroke stage with three-year mortality in patients with first-ever ischemic stroke. Cerebrovasc Dis. 2012;34(1):55-62.

32. Mazza A, Pessina A, Pavei A, Scarpa R, Tikhonoff V, Casiglia E. Predictors of stroke mortality in elderly people from the general population: the cardiovascular study in the elderly. Eur J Epidemiol. 2001;17(12):1097-104.

33. Gariballa S, Robinson T, Fotherlay M. Hypokalaemia and potassium excretion in stroke patients. J Am Geriator Soc. 1997;45(12):1454-8.

\section{Publisher's Note}

Springer Nature remains neutral with regard to jurisdictional claims in published maps and institutional affiliations.

\section{Submit your manuscript to a SpringerOpen ${ }^{\circ}$ journal and benefit from:}

- Convenient online submission

- Rigorous peer review

- Open access: articles freely available online

- High visibility within the field

- Retaining the copyright to your article

Submit your next manuscript at $\boldsymbol{\nabla}$ springeropen.com 